\title{
O PERFIL DO GESTOR ESPORTIVO BRASILEIRO: REVISÃO SISTEMÁTICA DA LITERATURA
}

\author{
THE PROFILE OF BRAZILIAN SPORTS MANAGERS: A SYSTEMATIC \\ LITERATURE REVIEW
}

\section{EL PERFIL DEL GESTOR DEPORTIVO BRASILEÑO: REVISIÓN BIBLIOGRÁFICA SISTEMÁTICA}

\author{
Thaís Camargo Zanatta*, Daiane Miranda de Freitas*, Filipe Gomide Carelli**, \\ Israel Teoldo da Costa*
}

\begin{abstract}
Palavras chave: Organização e Administração. Gestão da Qualidade. Gerência. Literatura de Revisão como Assunto.

Resumo: Este estudo teve como objetivo analisar os perfis dos gestores esportivos brasileiros apresentados pela literatura, no período de 1980 a 2016. Os trabalhos analisados foram obtidos em bases de dados eletrônicas e repositórios de universidades. As palavras-chave utilizadas foram "gestão esportiva", "gestão do esporte", "gestor esportivo", "dirigente esportivo", "administração esportiva" e "perfil e gestor esportivo". Com a utilização do método PRISMA, 19 estudos foram analisados. Esses trabalhos evidenciaram que o gestor esportivo brasileiro atua em organizações públicas, privadas e sem fins lucrativos e é, em sua maioria, do sexo masculino, com idade média de 42 anos, formação predominante em Educação Física e Administração e tempo de experiência máximo de 14 anos. Tais resultados apontam a necessidade de estratégias para a inserção da mulher no campo da gestão esportiva, além de enfatizar a importância de uma formação específica para os gestores esportivos brasileiros.
\end{abstract}

Keywords: Organization and Administration. Quality Management. Management. Literature Review as a Topic.

Palabras clave: Organización y Administración. Gestión de la Calidad. Gestión. Literatura de Revisión como Asunto.

\begin{abstract}
This study looked into the profiles of Brazilian sports managers presented in the literature from 1980 to 2016. The articles analyzed were obtained from electronic databases and university repositories. The keywords used were "sports management", "sports manager", "sports director" and "profile and sports manager" (in their Portuguese versions). Nineteen studies were examined by the PRISMA's method. They showed that Brazilian sports managers work in public, private and nonprofit organizations and are predominantly male. On average, they are 42 years old, predominantly trained in Physical Education and Business Administration, and have maximum work experience of 14 years. These results point out the need for strategies to include women in the field of sports management. In addition, they emphasize the importance of specific training for Brazilian sports managers.
\end{abstract}

Resumen: Este estudio tuvo como objetivo analizar los perfiles de los gestores deportivos brasileños presentados por la literatura en el período de 1980 a 2016. Los trabajos analizados se obtuvieron en bases de datos electrónicas y repositorios de universidades. Las palabras clave utilizadas fueron "gestión deportiva", "gestión del deporte", "gestor deportivo", "dirigente deportivo", "administración deportiva" y "perfil y gestor deportivo". gestor deportivo brasileño actúa en organizaciones públicas, privadas y sin fines de lucro y es, en su mayoría, del sexo masculino, con edad media de 42 años, formación predominante en Educación Física y Administración y con tiempo de experiencia máximo de 14 años. Estos resultados apuntan hacia la necesidad de estrategias para la inserción de la mujer en el campo de la gestión deportiva, además de enfatizar la importancia de una formación específica para los gestores deportivos brasileños. Con el método PRISMA, se analizaron 19 estudios. Estos trabajos evidencian que el
*Universidade Federal de Viçosa. Viçosa, MG, Brasil.

E-mail: efi.thaiscz@gmail.com; daianedefreitas@gmail.com; israelteoldocosta@gmail.com

**Universidade Federal de Juiz de Fora. Juiz de Fora, MG, Brasil.

E-mail: filipe.carelli@outlook.com

Recebido em: 26-06-2017 Aprovado em: 20-11-2017

DOI: http://dx.doi.org/10.22456/1982-8918.73803 (c) (1) (8) Licence 


\section{INTRODUÇÃO}

Desde o final da década de 60, nos Estados Unidos, a gestão esportiva tem sido notável em programas acadêmicos que foram criados para atender as demandas do esporte profissional e universitário. Acreditava-se que esses esportes poderiam ser melhor organizados se seus dirigentes tivessem o perfil e uma formação adequada que lhes permitissem propor melhorias organizacionais e, consequentemente, propiciar um melhor desenvolvimento econômico e estrutural desses esportes (PIRES; LOPES, 2001; ROCHA; BASTOS, 2011).

Essa iniciativa de propor formação específica aos gestores esportivos com o passar dos anos foi se ampliando e consolidando dentro das universidades ao redor do mundo, que passaram a desenvolver currículos cada vez mais especializados, com conteúdos aplicados ao contexto esportivo integrando as áreas de economia, finanças, marketing, comunicação, ética, legislação e política (PARKHOUSE, 1991; BASTOS, 2003).

Os programas de formação em gestão esportiva especificamente no Brasil começam a despontar a partir da década de 1970, como resultado da procura por profissionais que atendessem às demandas do mercado nacional (BASTOS, 2003). Mesmo com os avanços verificados ao longo das últimas décadas, os cursos de formação em gestão esportiva no país diferem-se dos programas oferecidos nos Estados Unidos e alguns países da Europa, principalmente em relação às propostas curriculares (CAPINUSSÚ, 2005; NOLASCO et al., 2005; MAZZEI et al., 2013). Os programas nacionais, como cursos de bacharelado e tecnológico, têm enfoques curriculares distintos, sendo o primeiro voltado para o ensino de disciplinas sobre fundamentos da gestão e o segundo direcionado aos aspectos culturais e de caracterização do esporte (MAZZEl et al., 2013).

A formação em gestão esportiva impulsionou a publicação de estudos científicos no Brasil, que se consolidaram ao longo dos anos com o estabelecimento de grupos de pesquisa e o delineamento de suas linhas de estudo (BASTOS, 2003; ROCHA; BASTOS, 2011). Assim, verifica-se que as universidades brasileiras passaram a partir da década de 1980 a estimular e desenvolver estudos científicos para subsidiar ações integradas entre o setor acadêmico e a práxis. Enquanto no cenário internacional universidades direcionam suas pesquisas para as áreas de gestão e liderança e marketing do esporte, no Brasil os estudos concentram-se nos aspectos de gestão e liderança e têm como um dos principais objetivos identificar o perfil dos gestores esportivos e destacar a importância da sua capacitação, para o sucesso das organizações esportivas (ROCHA; BASTOS, 2011; BASTOS, 2003).

Atualmente, um dos desafios para a área de gestão esportiva é a criação de cursos com estruturas curriculares que reúnam conhecimentos específicos sobre gestão e as diferentes concepções do esporte, considerando os aspectos científicos da área e as demandas do mercado, para promover assim a formação de profissionais com o perfil adequado para ocupar cargos de gestão nas diferentes organizações esportivas (MAZZEI et al., 2013; FREITAS, 2015).

Nesse sentido, a identificação do perfil dos gestores esportivos torna-se importante para traçarmos um panorama de quem está à frente das organizações esportivas e, consequentemente, compreendermos os aspectos essenciais da sua atuação, de forma que se possa verificar quais são as limitações e as potencialidades dos cursos/formações pelos quais esses gestores passaram e, assim, propor melhorias em pontos críticos da qualificação, 
assim como explorar melhor as potencialidades presentes nesses profissionais. Portanto, este artigo tem como objetivo analisar o perfil dos gestores esportivos brasileiros, no que tange ao sexo, idade, formação, tempo de experiência e vida esportiva, tendo como base a literatura nacional e internacional.

\section{METODOLOGIA}

Este trabalho caracteriza-se como uma revisão sistemática conduzida de acordo com o método PRISMA - Preferred Reportingltems for Systematic Reviews and Meta-Analysis (MOHRER et al. 2009; LIBERATI et al. 2009).

Após a exclusão dos estudos duplicados, 82 foram selecionados para a leitura pois apresentavam em seu título ou resumo ao menos uma das palavras-chave definidas pelos pesquisadores. Os respectivos documentos foram submetidos a uma triagem inicial considerando os seguintes aspectos: título e resumo, características da amostra, variáveis analisadas e a relevância dos resultados, o que resultou na eliminação de 49 estudos. Os documentos selecionados passaram por uma triagem final pela leitura completa com o intuito de verificar se atendiam aos critérios de inclusão: (1) estudos que discorressem sobre o perfil do gestor esportivo brasileiro e que fossem monografias, dissertações, teses, ou artigos, (2) documentos publicados no período de 1980 a 2016, (3) documentos publicados em português, inglês ou espanhol. Consideraram-se os seguintes critérios de exclusão: artigos de revisão e estudos que não apresentaram abordagem científica específica do perfil do gestor esportivo brasileiro.

Figura 1 - Fluxograma de seleção dos documentos selecionados, segundo a recomendação PRISMA.

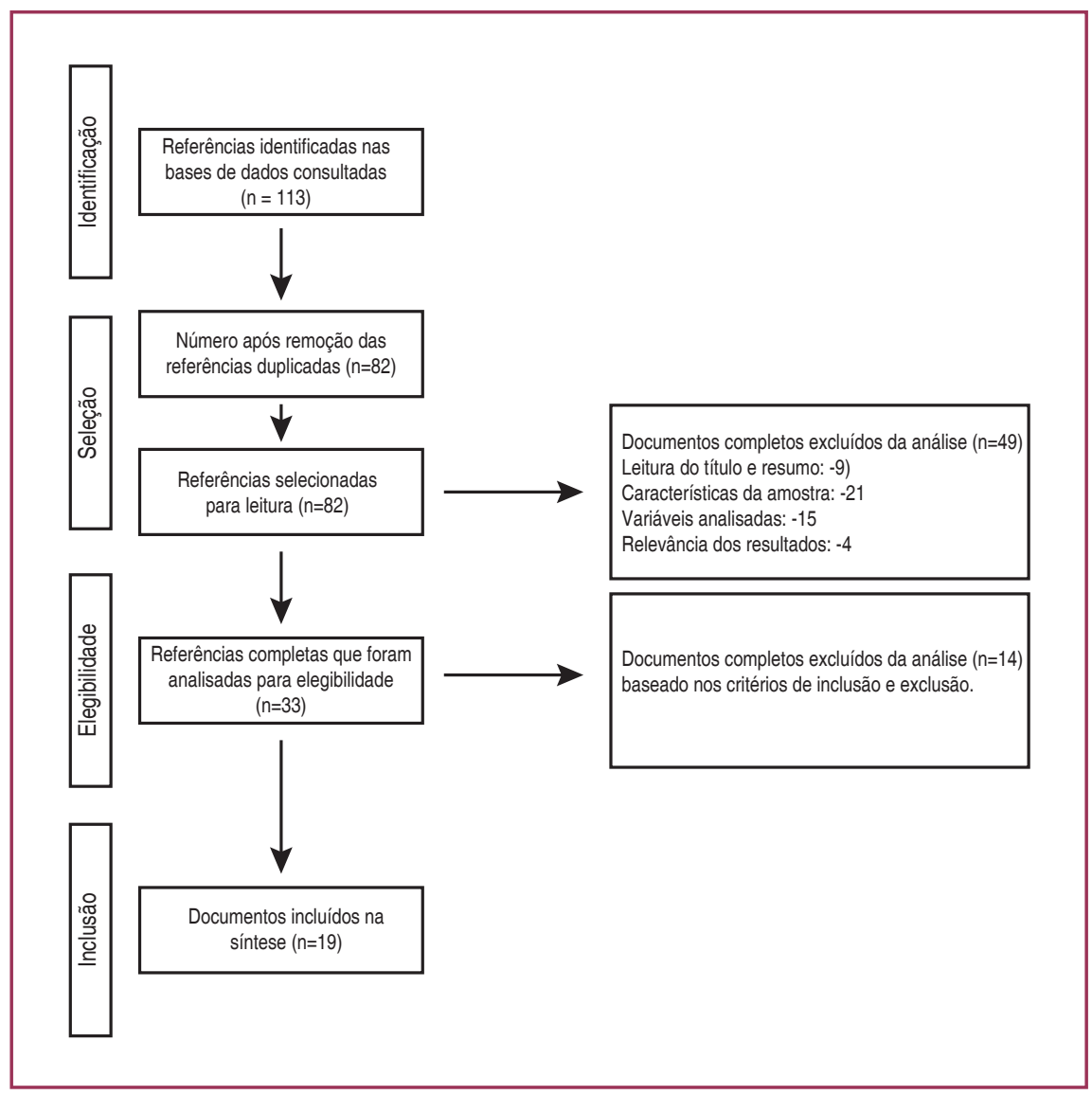


As palavras-chave utilizadas para a pesquisa foram "gestão do esporte", "gestor esportivo", "dirigente esportivo", "administração esportiva" e "perfil e gestor esportivo" e respectivas palavras análogas nas línguas inglesa e espanhola.

As pesquisas foram realizadas nas seguintes bases de dados: EBSCO, Academic Search Complete, Business Source Complete, Fonte Acadêmica, ScientificElectronic Library Online (ScIELO), SCOPUS, Portal Periódicos CAPES, Repositório Aberto da Universidade do Porto (UP), Repositório Aberto da Universidade Técnica de Lisboa (UTL), Repositório Universidade Trás-os-Montes e Alto Douro (UTAD), Repositório Institucional da Universidade Federal do Rio Grande do Sul (UFRGS), Repositório Digital da Fundação Getulio Vargas e Google Acadêmico. Realizou-se uma busca reversa considerando as referências dos estudos selecionados para complementar a respectiva seleção de documentos.

\section{RESULTADOS E DISCUSSÃO}

Os estudos sobre o perfil dos gestores esportivos brasileiros foram organizados no Quadro 1 conforme as seguintes categorias de análise: local de realização dos estudos, ano de publicação, autores e principais resultados.

Tendo em vista os critérios de inclusão e exclusão, 19 estudos foram selecionados, sendo 11 artigos advindos de periódicos, quatro publicações em anais de congresso, uma monografia e três dissertações. As pesquisas foram classificadas com base nos estados brasileiros, devido às suas dimensões territoriais e as características culturais presentes nas respectivas regiões.

Quadro 1 - Dados dos estudos que identificaram os perfis dos gestores esportivos brasileiros.

\begin{tabular}{|c|c|c|c|}
\hline Local & Ano & Autores & Principais resultados \\
\hline $\begin{array}{l}\text { Distrito } \\
\text { Federal }\end{array}$ & 2004 & $\begin{array}{c}\text { Azevêdo, P. H.; } \\
\text { Barros, J. F. }\end{array}$ & $\begin{array}{l}\text { Os técnicos que atuaram em órgãos governamentais avaliados } \\
\text { foram classificados, em sua maioria, como mulheres, com idade } \\
\text { média de } 42 \text { anos, aproximadamente } 14 \text { anos de experiência, } \\
\text { graduação em Educação Física, pós-graduação latu sensu } \\
\text { completa e renda média de } 12 \text { salários mínimos. }\end{array}$ \\
\hline $\begin{array}{l}\text { Distrito } \\
\text { Federal }\end{array}$ & 2004 & $\begin{array}{l}\text { Azevêdo, P. H.; } \\
\text { Barros, J. F.; } \\
\text { Suaiden, S. }\end{array}$ & $\begin{array}{l}\text { Dirigentes de clubes de futebol do Distrito Federal foram } \\
\text { caracterizados como homens católicos, casados, com idade média } \\
\text { de } 45,5 \text { anos e ensino fundamental completo; funcionários públicos } \\
\text { ou empresários, com renda média de } 25 \text { salários mínimos. }\end{array}$ \\
\hline São Paulo & 2006 & $\begin{array}{l}\text { Bastos, F. C. } \\
\text { et al. }\end{array}$ & $\begin{array}{l}\text { Administradores de clubes socioculturais e esportivos avaliados } \\
\text { foram identificados como homens, casados, com idade entre } \\
40 \text { e } 49 \text { anos, formação específica em Educação Física e } \\
\text { especialização, atuantes nas áreas recreativa, competitiva e de } \\
\text { formação esportiva. }\end{array}$ \\
\hline $\begin{array}{c}\text { Abrangência } \\
\text { Nacional }\end{array}$ & 2006 & $\begin{array}{c}\text { Gomes, E. M. } \\
\text { P.; } \\
\text { Mourão, L. }\end{array}$ & $\begin{array}{l}\text { A maioria das presidentes e vice-presidentes de federações } \\
\text { e confederações brasileiras entrevistadas não têm filhos, são } \\
\text { solteiras ou divorciadas, com graduação em Educação Física } \\
\text { e especialização na área de Gestão. Parte da amostra tem } \\
\text { experiência prévia como atleta de alto rendimento. }\end{array}$ \\
\hline $\begin{array}{l}\text { Distrito } \\
\text { Federal }\end{array}$ & 2009 & $\begin{array}{l}\text { Azevêdo, P. H.; } \\
\text { Spessoto, R. } \\
\text { E. N. }\end{array}$ & $\begin{array}{l}\text { Os dirigentes máximos das organizações futebolística foram } \\
\text { classificados como homens, casados, com idade variando de } \\
46 \text { a } 55 \text { anos, funcionários públicos, que ocupam a função de } \\
\text { presidente em suas respectivas organizações, recebendo salários } \\
\text { com valores que variam entre } 1.422,64 \text { e 1.778,31 dólares. }\end{array}$ \\
\hline
\end{tabular}


Continuação do quadro $1 . .$.

\begin{tabular}{|c|c|c|c|}
\hline Amazonas & 2010 & Anchieta, T. & $\begin{array}{l}\text { Gestores esportivos de instituições públicas do Amazonas foram } \\
\text { classificados como homens, com idade média de } 42 \text { anos e } \\
\text { ensino superior completo. Esses gestores consideram-se hábeis, } \\
\text { pacientes e cooperativos, porém, reconhecem a necessidade de } \\
\text { aperfeiçoamento quanto à competência administrativa, pois julgam } \\
\text { tal competência essencial na gestão desportiva. }\end{array}$ \\
\hline $\begin{array}{c}\text { Abrangência } \\
\text { Nacional }\end{array}$ & 2010 & $\begin{array}{l}\text { Maroni, F. C.; } \\
\text { Mendes, D. R.; } \\
\text { Bastos, F. C. }\end{array}$ & $\begin{array}{c}\text { A maioria dos gestores de equipes de voleibol foram identificados } \\
\text { como homens, com idade entre } 30 \text { e } 59 \text { anos, com atividades } \\
\text { profissionais além da gestão de suas equipes. Esses gestores } \\
\text { não possuem experiência como atleta, porém, possuem formação } \\
\text { superior sendo Educação Física e Administração os cursos mais } \\
\text { registrados. }\end{array}$ \\
\hline São Paulo & 2010 & $\begin{array}{l}\text { Palma, D.; } \\
\text { Inácio, S. L. }\end{array}$ & $\begin{array}{l}\text { O estudo verificou que gestores de organizações que representam } \\
\text { o esporte universitário paulista, atuam em Associações Atléticas } \\
\text { (84\%) e Departamentos de Esportes (15\%) e têm idade média de } \\
22 \text { e } 41 \text { anos, respectivamente; exercendo funções de presidente, } \\
\text { vice-presidente, coordenador e diretor; sendo que } 60,3 \% \text { têm } \\
\text { experiência com modalidades esportivas e } 26 \% \text { têm graduação } \\
\text { superior completa. }\end{array}$ \\
\hline Pernambuco & 2010 & $\begin{array}{c}\text { Pedroso, C. A. } \\
\text { M. Q. et al. }\end{array}$ & $\begin{array}{c}\text { Dirigentes das federações esportivas de Pernambuco foram } \\
\text { identificados como homens, com idade entre } 41 \text { e } 49 \text { anos e curso } \\
\text { superior completo (50\%). }\end{array}$ \\
\hline Amazonas & 2010 & $\begin{array}{l}\text { Silva, Z. C.; } \\
\text { Netto, S. }\end{array}$ & $\begin{array}{l}\text { Os gestores atuantes nos centros esportivos de Manaus foram } \\
\text { caracterizados como homens ( } 57,14 \%) \text { e mulheres }(42,85 \%) \\
\text { professores de Educação Física; } 43 \% \text { dos centros esportivos } \\
\text { avaliados realizam o planejamento por meio de coordenadores e } \\
\text { professores. }\end{array}$ \\
\hline $\begin{array}{c}\text { Abrangência } \\
\text { Nacional }\end{array}$ & 2011 & $\begin{array}{l}\text { Bastos, F. C.; } \\
\text { Fagnani, E. K.; } \\
\text { Mazzei, L. C. }\end{array}$ & $\begin{array}{l}\text { Os gestores das academias avaliadas foram classificados com } \\
\text { mulheres que têm experiência de aproximadamente seis anos } \\
\text { como gestoras de academia de fitness, graduadas em Educação } \\
\text { Física com especialização nas áreas de administração ou } \\
\text { marketing. }\end{array}$ \\
\hline Minas Gerais & 2011 & $\begin{array}{l}\text { Costa, L. G. L.; } \\
\text { Carvalho, A. S.; } \\
\text { Santos, R. C. } \\
\text { P. S. }\end{array}$ & $\begin{array}{c}\text { Os dirigentes das federações mineiras entrevistados foram } \\
\text { identificados com homens }(90,5 \%) \text {, com idade média de } 57 \text { anos } \\
\text { e curso superior completo }(80 \%) \text {. Essas federações apresentam } \\
\text { planejamento estratégico. }\end{array}$ \\
\hline Minas Gerais & 2011 & $\begin{array}{l}\text { Couto, A. C. P. } \\
\text { et al. }\end{array}$ & $\begin{array}{l}\text { Gestores esportivos atuantes em instituições públicas, privadas e } \\
\text { sem fins lucrativos da região metropolitana de Belo Horizonte foram } \\
\text { identificados como sendo do sexo masculino ( } 76,6 \%) \text {, atuantes no } \\
\text { setor privado e com idade média de aproximadamente } 38 \text { anos. }\end{array}$ \\
\hline Rio de Janeiro & 2011 & $\begin{array}{l}\text { Santos, A. R.; } \\
\text { Franco, P. V. } \\
\text { D. }\end{array}$ & $\begin{array}{l}\text { Os gestores das academias do Rio de Janeiro foram classificados } \\
\text { como profissionais jovens, inovadores e criativos, solteiros, com } \\
\text { formação em Educação Física e especialização na área de gestão } \\
\text { de pessoas. }\end{array}$ \\
\hline Amazonas & 2011 & Valente, L. & $\begin{array}{l}\text { A maioria dos gestores de centros esportivos e de lazer de } \\
\text { Manaus foi caracterizada como mulheres, com idade entre } 40 \\
\text { a } 49 \text { anos e formação em Educação Física. Esses gestores } \\
\text { exercem suas funções há mais de um ano, dedicam-se mais às } \\
\text { tarefas relacionadas a direção e organização e têm experiências } \\
\text { como atletas. }\end{array}$ \\
\hline $\begin{array}{c}\text { Abrangência } \\
\text { Nacional }\end{array}$ & 2012 & $\begin{array}{c}\text { Santana, L. C. } \\
\text { et al. }\end{array}$ & $\begin{array}{l}\text { O perfil de gestores de academia fitness do Brasil constitui-se } \\
\text { de homens ( } 68,65 \% \text { ) e mulheres ( } 31,35 \%) \text {, com faixa etária } \\
\text { predominante entre } 30 \text { e } 39 \text { anos, sendo que } 90 \% \text { dos avaliados } \\
\text { têm curso superior completo. O tempo de experiência desses } \\
\text { gestores em seus respectivos cargos varia de } 1 \text { a } 10 \text { anos. }\end{array}$ \\
\hline
\end{tabular}


Continuação do quadro $1 . .$.

\begin{tabular}{|c|c|c|c|}
\hline $\begin{array}{c}\text { Abrangência } \\
\text { Nacional }\end{array}$ & 2013 & $\begin{array}{c}\text { Gestores de organizações esportivas participantes da Liga } \\
\text { Basquete Feminino foram classificados como homens, com idade } \\
\text { entre } 40 \text { e } 60 \text { anos, com ensino superior completo, com formação } \\
\text { predominante em Educação Física; 50\% têm especialização ou } \\
\text { pás-graduação e } 62,5 \% \text { exercem atividades paralelas a gestão } \\
\text { das organizações. Porém, esses profissionais não têm experiência } \\
\text { como atletas profissionais de basquete. }\end{array}$ \\
\hline $\begin{array}{c}\text { Rio Grande } \\
\text { do Sul }\end{array}$ & 2013 & Karnas, G. S. & $\begin{array}{r}\text { Os gestores esportivos dos municípios do Rio Grande do Sul } \\
\text { foram identificados como homens, com idade média de } 37 \text { anos e } \\
\text { graduação em Educação Física, com função de coordenadores ou } \\
\text { diretores, eles exercem atividades de planejamento, coordenação, } \\
\text { direção e organização. }\end{array}$ \\
\hline São Paulo & 2016 & $\begin{array}{c}\text { Amaral, C. M. } \\
\text { S.; } \\
\text { Bastos, F. C. }\end{array}$ & $\begin{array}{c}\text { Gestores de instalações esportivas de São Paulo avaliados no } \\
\text { estudo foram classificados como homens, com faixa etária entre } \\
50 \text { e } 59 \text { anos, graduados em Educação Física e com até dois anos } \\
\text { de experiência no cargo. As funções exercidas por esses gestores } \\
\text { são diversificadas, destacando-se a função de coordenador. }\end{array}$ \\
\hline
\end{tabular}

Fonte: Autores.

Com os resultados obtidos percebe-se que os estudos de caracterização do gestor esportivo brasileiro são datados efetivamente a partir de 2004. Esse resultado corrobora a história da implantação da área no contexto acadêmico e criação de grupos e centros de pesquisas, como, por exemplo, os grupos constituídos na USP, UFPA, UNB, UFSC a partir da década de 2000 (BASTOS; MAZZEI, 2012).

No Brasil, Rezende (2000) identifica as organizações esportivas sob a ótica da organização como unidade social: organizações que existem em função da atividade física, esporte e lazer, como centros de treinamento e escolinhas; academias; clubes e associações exclusivamente esportivas; consultorias e assessorias; ligas, federações e confederações; fundações, instituições e comitês, entre outros; e aquelas que promovem o esporte através de seus setores/departamentos esportivos específicos como prefeituras, governos estaduais, governo federal, clubes sociais, entidades representativas (SESC, SESI, sindicatos), entre outras.

Os 19 estudos analisados corresponderam à pesquisa com gestores esportivos atuantes na administração pública e privada (AZEVÊDO; BARROS, 2004; ANCHIETA, 2010; PALMA, 2010; KARNAS, 2013; SILVA; NETO, 2010; AMARAL; BASTOS, 2016; VALENTE, 2011), federações e confederações esportivas (GOMES; MOURÃO, 2006; COSTA; CARVALHO; SANTOS, 2011; PEDROSO et al., 2010), academias (BASTOS; FRAGNANI; MAZZEI, 2011; SANTOS; FRANCO, 2011; SANTANA; MONTEIRO; PEREIRA; BASTOS, 2012), equipes e clubes esportivos (MARONI; MENDES; BASTOS, 2010; AZEVÊDO; SPESSOTO, 2009; AZEVÊDO; BARROS; SUAIDEN, 2004; BASTOS et al., 2006; GITTI; BASTOS, 2013) e um estudo que envolveu instituições sem fins lucrativos, públicas e privadas (COUTO et al., 2011).

Considerando os locais de realização dos estudos, destaca-se o número maior de publicações na Região Sudeste, resultando em seis estudos. Três pesquisas caracterizaram o perfil dos gestores esportivos na capital de São Paulo, sendo um estudo realizado nos clubes socioculturais e esportivos (BASTOS et al., 2006), outro no esporte universitário (PALMA; 
INÁCIO, 2010) e, por fim, um estudo com gestores de instalações esportivas públicas e privadas (AMARAL; BASTOS, 2016). No Rio de Janeiro um estudo caracterizou os gestores de academias (SANTOS; FRANCO, 2011) e, em Minas Gerais, um estudo foi realizado com os presidentes e vice-presidentes de federações esportivas (COSTA; CARVALHO; SANTOS, 2011) e outro estudo buscou identificar o perfil dos gestores de instituições de capital privado, públicas e sem fins lucrativos da região metropolitana de Belo Horizonte (COUTO et al., 2011).

$\mathrm{Na}$ Região Central do Brasil três pesquisas foram realizadas em Brasília, sendo uma pesquisa realizada com gestores que atuaram no planejamento e controle de projetos esportivos realizados pelo governo federal durante o período de 1995 a 2002 (AZEVÊDO; BARROS, 2004) e outros dois estudos com foco em dirigentes de clubes de futebol (AZEVÊDO; BARROS; SUAIDEN, 2004; AZEVÊDO; SPESSOTO, 2009).

No Norte do Brasil três estudos ocorreram em Manaus, capital do Amazonas, identificando o perfil dos gestores públicos do Centro de Esporte e Lazer (VALENTE, 2011; SILVA; NETTO, 2010) e outro caracterizando os secretários do esporte municipal e estadual, bem como os presidentes das federações esportivas do estado do Amazonas (ANCHIETA, 2010).

No Nordeste e Sul do país confirmou-se apenas uma pesquisa em cada região com os presidentes das federações esportivas de Pernambuco (PEDROSO et al., 2010) e com os gestores de organizações públicas do Rio Grande do Sul (KARNAS, 2013), respectivamente.

$E$, finalmente, cinco pesquisas foram realizadas com abrangência de diferentes unidades federativas brasileiras, incluindo pesquisas com gestores de academias (BASTOS; FAGNANI; MAZZEI, 2011; SANTANA et al., 2012), gestores de clubes de voleibol (MARONI; MENDES; BASTOS, 2010), presidentes e vice-presidentes de federações e confederações (GOMES; MOURÃO, 2006) e gestores de equipes participantes da Liga Basquete Feminino (GITTI; BASTOS, 2013).

De acordo com o resultado, verificou-se que as maiores amostras são provenientes de uma pesquisa realizada com os gestores esportivos atuantes em academias de vinte estados brasileiros (SANTANA et al., 2012), com 263 participantes; e com os gestores esportivos atuantes nas prefeituras dos municípios do Rio Grande do Sul (KARNAS, 2013), com 169 participantes.

Ao analisar os materiais e métodos, observou-se que todos os estudos se assemelham quanto aos fins da pesquisa e quanto ao instrumento para coleta de dados, destacando a pesquisa quantitativa e a aplicação do questionário, respectivamente.

Pesquisas neste campo da gestão em que se busca definir o perfil, formação, competências e funções do gestor esportivo têm evidenciado a metodologia quantitativa, conforme constatado na revisão da literatura realizada por Joaquim, Batista e Carvalho (2011). Em alguns casos, como Azevêdo e Barros (2004), a finalidade da pesquisa se caracteriza como quantitativo-qualitativa, que para além da aplicação dos questionários utilizaram-se entrevistas e documentos, em decorrência dos objetivos propostos.

\subsection{Análise do perfil do gestor}

A seguir apresentam-se os resultados do perfil dos gestores esportivos brasileiros quanto a sexo, idade, formação, tempo de experiência e vida esportiva. 


\subsubsection{Sexo}

As amostras de 11 estudos foram constituídas por indivíduos de ambos os sexos, sendo sete com maioria masculina e quatro com maioria feminina, sete estudos resultaram em participantes exclusivamente masculinos e um estudo resultou em participantes femininos. Este estudo, especificamente com gestoras, refere-se à investigação de Gomes e Mourão (2006) com dez participantes (presidentes e vice-presidentes de federações e confederações brasileiras) que buscou compreender as formas de inserção das dirigentes e construir um perfil das gestoras esportivas nacionais.

No Brasil, conforme afirma Gomes (2005), a participação feminina no Ministério dos Esportes ocorre em sua maioria em cargos de assessoria e não nos cargos de chefia, coordenação ou diretoria, sendo representada por 20,45\%. Ainda segundo a autora, quando considerados os cargos de presidente em federações esportivas brasileiras, em 2004 somente $7 \%$ eram presididas por mulheres, sendo a maioria nas federações de Ginástica Olímpica.

Em outro estudo, Gomes e Mourão (2006) retratam a distribuição das mulheres na gestão das confederações e federações brasileiras resultando, respectivamente, em 1,8\% e $6,5 \%$ de mulheres. Contudo, verificou-se que a desigualdade da participação feminina em cargos de direção de organizações esportivas vem sendo retratada em diversas pesquisas, em diversos países.

O baixo índice de representação das mulheres em posições administrativas também é verificado quando se trata de organizações de nível olímpico, como o Comitê Olímpico Internacional (COI), com 5\% (MIRAGAYA, 2002), e Comitê Olímpico Brasileiro (COB), com cerca de 4\% de mulheres (GOMES, 2005; OLIVEIRA; TEIXEIRA, 2009), além das federações esportivas nacionais, com 6,5\% de participação feminina (GOMES, 2005). Diante dessa realidade, já constatada há muitos anos, o Comitê Olímpico Internacional, a partir de seus Comitês Nacionais, decidiu por fazer a implementação de políticas de incentivo para inserção de mulheres na gestão esportiva, tornando-se um incentivo à inclusão das mulheres no universo esportivo (GOELLNER, 2004). Entretanto, não é isso que, ainda nos dias atuais, constatamos. Essa realidade não é apenas no mundo esportivo, é também o que se presencia em diferentes organizações, como hospitais, forças armadas e nas esferas do poder público (SILVA et al., 2016; INTER-PARLAMENTARY UNION, 2015; MATOS, et al., 2016).

\subsubsection{Idade}

A idade dos gestores esportivos foi identificada por 18 estudos, sendo que seus autores apresentam os resultados de forma distinta, seja em intervalos ou em média de idades. Os estudos de Gomes e Mourão (2006), Maroni, Mendes e Bastos (2010), Bastos, Fagnani e Mazzei (2011), Azevêdo e Spessoto (2009), Pedroso, Sarmento, Albuquerque (2010), Valente (2011), Santos e Franco (2011), Bastos et al. (2006), Santana et al. (2012), Amaral e Bastos (2016), Silva e Netto (2010), Gitti e Bastos (2013) e Karnas (2013) apresentaram os resultados em intervalos que variam entre 20 e 66 anos.

Os menores intervalos de idade foram encontrados no estudo de Pedroso, Sarmento e Albuquerque (2010), com variação da idade de 41 a 49 anos. Já o maior intervalo encontrado foi no estudo de Karnas (2013), com gestores que apresentaram idades entre 20 e 66 anos. A partir desses dados nota-se que a faixa etária predominante entre os gestores é de 40 a 49 anos. 
Entre os estudos que classificaram a idade dos gestores esportivos pela média, verificou-se o mínimo de 32,8 anos (COUTO et al., 2011) e máximo de 57 anos (COSTA; CARVALHO; SANTOS, 2011). Considerando todos os estudos que apresentaram a média de idades houve uma maior incidência de gestores com 42 anos. Corroborando estes dados, na pesquisa de Gomes e Mourão (2006), as gestoras das federações e confederações brasileiras têm a média de idade equivalente a 40 anos.

\subsubsection{Formação}

Dentre as pesquisas analisadas que identificam a formação dos gestores, verificou-se que a graduação em Educação Física é o principal curso de graduação dos gestores esportivos. Entretanto, nos estudos de Gomes et al. (2004), Anchieta (2010) e Amaral e Bastos (2016), os gestores esportivos são oriundos de outras áreas, como, por exemplo, Administração, Fisioterapia, Design, Engenharia, Psicologia, Agronomia, Pedagogia, entre outras.

A discussão acerca da formação inicial necessária para intervir na gestão esportiva tem sido foco de muitos pesquisadores nos últimos anos. Sarmento, Pinto e Oliveira (2006), Bastos (2003) e Pires e Lopes (2001) acreditam que a formação deve basear-se em conhecimentos multidisciplinares adequados à realidade da área, porém, para estes últimos autores é indispensável uma formação inicial em gestão do esporte. 0 estudo de Freitas et al. (2015) mostrou que mesmo julgando-se competente quanto às suas funções de liderança gerencial os presidentes de federações olímpicas brasileiras reconhecem a necessidade da atualização de suas competências, além de destacar a aquisição de certificação em diferentes áreas de conhecimento e a obtenção de experiências esportivas e gerenciais como ferramentas importantes para a solução das demandas de suas instituições.

A gestão do esporte é um campo de intervenção de diversas especialidades, por isso, como afirma Pires e Lopes (2001, p. 98) "[...] não pode ser analisada nem compreendida e avaliada, a não ser considerando todas as contribuições técnicas científicas, sociais e pedagógicas do quadro social específico em que está a ser aplicada".

Ressalta-se a partir dessa discussão que o gestor esportivo deverá ter sempre em mente as funções de um gestor no seu conceito lato, que, conforme descreve Mintzberg (1975),é aquele que desempenha competências relacionadas às relações interpessoais, processos informacionais e tomadas de decisão. Desta forma, o que se espera do gestor na sua atuação é a aplicação destas competências gerenciais, o que muitas vezes não está presente nos currículos de formação de uma forma geral, conforme foi visto nesta pesquisa e reportado por outros estudos.

\subsubsection{Tempo de experiência}

Dos 19 estudos analisados, essa variável foi caracterizada em 11, sendo apresentada em intervalos que variam de meses a anos de experiência. O estudo de Karnas (2013), com 169 participantes, apresentou o menor valor para o tempo de experiência, em um intervalo que identificou valores menores que um ano para os gestores esportivos dos municípios do Rio Grande do Sul. Já o estudo de Azevêdo e Barros (2004) apresentou o maior valor dentre os nove estudos que avaliaram o tempo de experiência dos gestores esportivos, 14 anos de experiência. 
No setor público a média do tempo de experiência dos gestores é maior que no setor privado, sendo 14 e 10 anos, respectivamente, considerando as maiores médias identificadas nas investigações.

Os dois estudos que pesquisaram os presidentes de federações esportivas identificaram os seus tempos de experiência. O estudo realizado por Costa, Carvalho e Santos (2011) apresentou um intervalo que varia de um a quatro anos de experiência para os presidentes e vice-presidentes de federações esportivas mineiras. Em Pernambuco, os gestores de federações olímpicas têmuma experiência no cargo/função variando entre três e cinco anos (PEDROSO et al., 2010).

O tempo de experiência foi considerado por presidentes de federações olímpicas brasileiras um aspecto essencial para o desenvolvimento de articulações políticas e a aquisição de conhecimentos técnicos e organizacionais que, além de possibilitar o fortalecimento nas relações presidente-membros, viabiliza a comunicação e a execução de tarefas (FREITAS, et al., 2016). Além disso, esses presidentes classificaram o tempo de experiência como um recurso essencial para enfrentar melhor os desafios estratégicos e financeiros do trabalho (FREITAS; GIRGINOV; TEOLDO, 2016).

O que se percebe quanto ao tempo de experiência no cargo é que com o passar do tempo os gestores ganham experiência, compreendem melhor toda a dinâmica organizacional, em especial os aspectos gerenciais, e desenvolvem habilidades requeridas pelo trabalho, especificamente nas organizações esportivas, neste caso.

\subsubsection{Vida esportiva}

Em relação à vida esportiva, apenas oito estudos identificaram essa variável (GOMES; MOURÃO, 2006; BASTOS et al., 2006; COSTA; CARVALHO; SANTOS, 2011; ANCHIETA, 2010; MARONI; MENDES; BASTOS, 2010; VALENTE, 2011; GITTI; BASTOS, 2013; KARNAS, 2013).Essas investigações, que incluem diferentes tipos de organizações, e diferente perfil dosparticipantes, identificaram seus gestores como ex-praticantes de modalidades esportivas, seja na totalidade ou na maioria da amostra, com exceção do estudo realizado com gestores (gerentes e supervisores) dos clubes de voleibol do Brasil, que mostrou que a maioria dos indivíduos não foi atleta (MARONI; MENDES; BASTOS, 2010).

Bastos et al. (2006) e Maroni, Mendes e Bastos (2010) pesquisaram a possível influência da experiência como atleta ser relevante na escolha e/ou capacitação do gestor, e o resultado obtido por Maroni, Mendes e Bastos (2010), equivalente a 36,4\% da amostra, corrobora os achados de Bastos e colaboradores (2006) em clubes socioculturais e esportivos, onde $28,57 \%$ são ex-atletas. Para alguns autores "[...] esses resultados sugerem que esta variável não é determinante no acesso do gestor ao cargo" (MARONI; MENDES; BASTOS, 2010 , p. 243). Porém, a experiência como praticante ou atleta de modalidades esportivas oferece aos gestores a oportunidade de compreender as especificidades do esporte, como, por exemplo, sua inserção na sociedade e técnicas específicas.

Apesar da vivência esportiva possibilitar diferentes experiências na modalidade praticada, assim como também se constata em outras áreas, como, por exemplo, na enfermagem (SILVA et al., 2016), e, consequentemente, favorecer práticas de liderança e 
gestão, esta experiência não dispensa a formação específica para a execução das funções e atuação enquanto gestores, através da qual se prepara o gestor em relação as competências gerenciais especificas.

\section{CONSIDERAÇÕES FINAIS}

Avanços significativos marcaram a história da gestão esportiva no Brasil. Dentre eles, destaca-se a publicação de investigações científicas que foram realizadas, entre outras finalidades, com o intuito de avaliar o perfil dos gestores esportivos brasileiros.

Nos 19 estudos analisados, sendo 11 artigos advindos de periódicos, quatro publicações em anais de congresso, uma monografia e três dissertações, verificou-se que os profissionais à frente das entidades esportivas no país são homens, com idade média de 42 anos, formação predominante em Educação Física e Administração e tempo de experiência de 14 anos. Além disso, esses gestores atuam em diferentes organizações públicas e privadas, como: associações, clubes, federações, academias de ginástica, órgãos de gestão pública federal, estadual e municipal e foram identificados em alguns estudos como ex-praticantes das modalidades esportivas que coordenam atualmente.

A predominância dos homens nos cargos de liderança das organizações esportivas brasileiras incita a reflexão sobre os obstáculos e diversidades enfrentados pelas mulheres que buscam oportunidades de atuação neste setor, reforçando ainda mais a importância das políticas de incentivo como uma estratégia para a inserção de gestoras brasileiras na área da gestão esportiva.

Por fim, vale ressaltar a necessidade da qualificação desses profissionais através de programas e especializações com matrizes curriculares voltadas especificamente para 0 ensino de conteúdos da área da gestão esportiva.

\section{REFERÊNCIAS}

AMARAL, Cacilda Mendes dos Santos; BASTOS, Flávia da Cunha. O perfil do gestor de instalações esportivas do município de São Paulo. Revista de Gestão e Negócios do Esporte, v. 1, n. 1, p. 50-63, maio 2016.

ANCHIETA, Tharcísio. Perfil do gestor esportivo no Amazonas. 2010. 111 f. Dissertação (Mestrado) - Faculdade de Desporto da Universidade do Porto, Porto, 2010.

AZEVÊDO, Paulo Henrique; BARROS, Jônatas de França. A necessidade de administração profissional do esporte brasileiro e o perfil do gestor público, em nível federal, que atuou de 1995 a 2002. Revista Digital - Lecturas Educación Física y Deportes, v. 10, n. 74, jul. 2004. Disponível em: <http://www.efdeportes.com/efd74/admin.htm>. Acesso em: 20 maio 2016.

AZEVÊDO, Paulo Henrique; BARROS, Jônatas de França; SUAIDEN, Samir. Caracterização do perfil do gestor esportivo dos clubes da primeira divisão de futebol do Distrito Federal e suas relações com a legislação esportiva brasileira. Revista de Educação Física/UEM, v.15, n.1, p. 33-42.jan. 2004. 
AZEVÊDO, Paulo Henrique; SPESSOTO, Rubens Eduardo Nascimento. Caracterização do perfil retrospectivo do dirigente esportivo de clube de futebol profissional da primeira divisão, entre os anos 2003 e 2007. Revista Portuguesa de Ciências do Desporto, v. 9, n. 2, p.103-112, 2009.

BASTOS, Flávia da Cunha. Administração Esportiva: área de estudo, pesquisa e perspectivas no Brasil. Motrivivência (UFSC), v. 15, n. 20/21, p.295-306, 2003.

BASTOS, Flávia da Cunha et al. Perfil do administrador esportivo de clubes sócio-culturais e esportivos de São Paulo/Brasil. Revista Mackenzie de Educação Física e Esportes, v. 5, n. 1, p.13-22, 2006.

BASTOS, Flávia Cunha; FAGNANI, Enrico Kaigawa; MAZZEI, Leandro Carlos. Perfil de gestores de redes de academia de fitness. Revista Mineira de Educação Física, v. 19, n. 1, p.64-74. 2011.

BASTOS, Flávia Cunha; MAZZEI, Leandro Carlos. Gestão do esporte no Brasil: desafios e perspectivas. In: MAZZEI, Leandro Carlos; BASTOS, Flávia Cunha. Gestão do esporte no Brasil: desafios e perspectivas. São Paulo: Ícone, 2012. p. 23-42.

CAPINUSSÚ, José Maurício. Formação de recursos humanos e a necessidade de uma administração desportiva moderna capaz de influir de forma positiva no desempenho da equipe e do atleta. Arquivos em Movimento, v.1, n.1, p.87-93. 2005.

COSTA, Lise Gouvêa Lisboa; CARVALHO, Amaylton Salles; SANTOS, Raquel Cristina Pereira de Souza. Perfil do gestor esportivo e diagnóstico da estrutura organizacional das Federações Mineiras. In: CONGRESSO ALGEDE, 2., 2011. CONGRESO DE LA ASOCIACIÓN LATINOAMERICANA DE LA GERENCIA DEPORTIVA, 2. [Anais...] Monterrey, 2011. p. 20-21.

COUTO, Ana Cláudia Porfirio et al. Perfil do gestor esportivo - análise centrada na região metropolitana de Belo Horizonte. In: CONGRESSO CIENTíFICO BRASILEIRO DA FIEP, 8., 2011. FIEP BULLETIN, v. 81, p 1-5, 2011.

FREITAS, Daiane Miranda. Competências de liderança dos presidentes das federações olímpicas brasileiras. 2015. 228 f. Tese (Doutorado) - Programa Doutoral em Ciências do Desporto, Faculdade de Desporto da Universidade do Porto, Porto, 2015.

FREITAS, Daiane Mirandaet al. Liderança dos presidentes das federações olímpicas brasileiras: Análise da autopercepção das competências em função da formação académica. Revista Portuguesa de Ciências do Desporto, v. 15, n. 1, p. 79-98, 2015.

FREITAS, Daiane Mirandaet al.Leadership ofPresidents of Brazilian Olympic Sport Federations: Comparison between Real and Ideal Perceived Competencies Profiles According to Their Experience. International Business Research, v. 9, n. 5, p. 146-157, 2016.

FREITAS, Daiane Miranda; GIRGINOV, Vassil; TEOLDO, Israel.What do they do? Competency and managing in Brazilian Olympic Sport Federations. European Sport Management Quarterly, v. 17, p. 1-17, 2016.

GITTI, Vivian da Silva; BASTOS, Flávia da Cunha. Estrutura Organizacional e Perfil do Gestor de Equipes Participantes da Liga Feminina de Basquete (LFB) 2011/2012. PODIUM: Sport, Leisure and Tourism Review, v. 2, n. 2, p. 53-75, jul./dez. 2013.

GOELLNER, Silvana Vilodre. Mulher, olimpismo e desempenho. In:FORUM DE DEBATES SOBRE MULHER \& ESPORTE, 3., 2004; FORUM INTERNACIONAL MULHER E ESPORTE, 1. , 2004. Anais...São Paulo: Aleph, 2004.p.42-46. 
GOMES, Euza Maria de Paiva. Esporte e Inclusão Social: Mulheres na gestão esportiva brasileira. In: DA COSTA, Lamartine. Atlas do Esporte no Brasil: atlas do esporte, educação física e atividades físicas de saúde e lazer no Brasil. Rio de Janeiro: Shape, 2005. p. 615-617.

GOMES, Euza Maria de Paiva; MOURÃO, Ludmila. As mulheres na gestão das federações esportivas no Brasil. In: MORAGAS, Miquel; DA COSTA, Lamartine. Seminários España-Brasil 2006. Barcelona:Rio de Janeiro: Universidade Autônoma de Barcelona, Universidade Gama Filho, 2006. p.72-81.

INTER-PARLAMENTARY UNION. New IPU and UM Women Map shows women's representation in politics stagnates. Disponível em: <https://www.ipu.org/news/pressreleases/2017-03/new-ipu-and-un-women-map-shows-womens-representation-in-politics-stagnates>. Acesso em: 19 out. 2017.

JOAQUIM, Bárbara Andreia; BATISTA, Paula Maria; CARVALHO, Maria José. Revisão Sistemática sobre o perfil de competências do gestor desportivo. Movimento, v. 17, n. 1, p. 255279, jan./mar. 2011.

KARNAS, Gabriel Silveira. Perfil do Gestor Esportivo dos Municípios do Rio Grande do Sul.2013.113 f.Dissertação (Mestrado) - Faculdade de Desporto da Universidade do Porto, Porto, 2013.

LIBERATI, Alessandro et al. The PRISMA Statement for Reporting Systematic Reviews and Meta-Analyses of Studies That Evaluate Health Care Interventions: Explanation and Elaboration. PLoSMed,v. 6, n. 7, jul. 2009. Disponível em: <https://doi.org/10.1371/journal.pmed.1000100>. Acesso em: 19 out. 2017.

MARONI, Fernando Castro; MENDES, DilsonRibau; BASTOS, Flávia da Cunha. Gestão do voleibol no Brasil: o caso das equipes participantes da Superliga 2007-2008. Revista Brasileira de Educação Física e Esportes, v. 24, n. 2, p. 239-48, abr./jun. 2010.

MATOS, Deise Justino et al. Mulheres nas Forças Armadas: Desenvolvimento Histórico-Jurídico da Participação Feminina na Defesa Nacional. In:CONGRESSO ACADÊMICO SOBRE DEFESA NACIONAL, 8., 2016. Trabalhos Acadêmicos; CONGRESSO ACADÊMICO SOBRE DEFESA NACIONAL (CADN), 13.,2016. [Anais...]. Rio de Janeiro, 2016. v. 1. p. 378-396.

MAZZEI, Leandro Carlos; AMAYA, Katherine; BASTOS, Flávia da Cunha. Programas acadêmicos de graduação em Gestão do Esporte no Brasil. Revista Mackenzie de Educação Física e Esporte, v.12, n.1, p.219-234. 2013.

MINTZBERG, Henry. The manager's job: folklore and fact. Harvard Business Review, v. 53, n. 4, p.49-61,1975.

MIRAGAYA, Ana. A mulher Olímpica: tradição versus inovação na busca pela inclusão. In: TURINI, Marcio; DA COSTA, Lamartine. Coletânea de textos em Estudos Olímpicos. Rio de Janeiro: Editora Gama Filho, 2002. p. 763-791.

MOHER, David et al. The PRISMA Group. Preferred reporting items for systematic reviews and meta-analyses: The PRISMA Statement. PLoSMed, v. 6, n. 7, jul. 2009. Disponível em: <https:// doi.org/10.1371/journal.pmed.1000097>. Acesso em: 19 out. 2017.

NOLASCO, Verônica Périssé et al. Administração/Gestão Esportiva. In: DA COSTA, Lamartine (Ed.). Atlas do esporte no Brasil: atlas do esporte, educação física e atividades físicas de saúde e lazer no Brasil. Rio de Janeiro: Shape, 2005. p. 760-761. 
OLIVEIRA, Gabriela Aragão Souza; TEIXEIRA, Ana Paula de Oliveira. Trilhando um novo caminho: a gestão esportiva. Revista Gênero, v. 10, n. 1, p.101-118, 2009.

PALMA, Deborah; INÁCIO, Sandra da Luz. Perfil dos gestores universitários da região metropolitana de São Paulo. Coleção Pesquisa em Educação Física, v. 9, n. 2, p. 215-222, 2010.

PARKHOUSE, Bonnie. The management of sport: its foundation and application. St. Louis: MosbyYear Book, 1991.

PEDROSO, Carlos Augusto Mulatinho Queirozet al. Perfil do gestor desportivo das federações olímpicas do Estado de Pernambuco. Revista Digital - Lecturas Educación Física y Deportes, v. 1, n. 145, jun. 2010. Disponível em: <http://www.efdeportes.com/efd145/perfil-do-gestordesportivo-das-federacoes-olimpicas.htm>. Acesso em: 12 jun. 2016.

PIRES, Gustavo Manuel Vaz da Silva; LOPES, José Pedro Sarmento de Rebocho. Conceitos de gestão do desporto. Novos desafios, diferentes soluções. Revista Portuguesa de Ciências do Desporto, v.1, n.1, p.88-103, 2001.

REZENDE, José Ricardo. Organização e administração no esporte. Rio de Janeiro: Sprint, 2000.

ROCHA, Cláudio Miranda; BASTOS, Flávia da Cunha. Gestão do Esporte: definindo a área. Revista Brasileira de Educação Física e Esporte, v. 25, n. Esp., p.91-103, dez. 2011.

SANTANA, Luis Carlos et al. O perfil de gestores de academia fitness no Brasil: um estudo exploratório. PODIUM: Sport, Leisure and Tourism Review, v. 1, n. 1, p.28-46, 2012.

SANTOS, Amanda Rodrigues; FRANCO, Paola Ventura Drummond. 0 perfil do gestor de academias de grande porte em diferentes bairros da cidade do Rio de Janeiro. 2011. $18 \mathrm{f}$. Trabalho de Conclusão de Curso (Programa de Pós-Graduação) - Universidade da Saúde, Rio de Janeiro, 2011.

SARMENTO, José Pedro; PINTO, Assunção; OLIVEIRA, André Esteves. O perfil organizacional e funcional do gestor desportivo em Portugal. Revista Portuguesa de Gestão de Desporto, v. 3 , n. 2, p. 58-63,2006.

SILVA, Vânea Lúcia dos Santos et al. Leadership practices in hospital nursing: a self of manager nurses. Revista da Escola de Enfermagem da USP, v. 207, n. 51, 2016. Disponível em: <http:// www.journals.usp.br/reeusp/article/view/129792/126390>. Acesso em: 19 out. 2017.

SILVA, Zulene Cordeiro; NETTO, Sidney.O Perfil do Gestor dos Centros Esportivos de Lazer Prefeitura Municipal de Manaus. FIEP BULLETIN, v. 80, Sp. Ed.,2010. Disponível em: <http:// fiepbulletin.net/index.php/fiepbulletin/article/view/902>. Acesso em: 12 nov. 2016.

VALENTE, Lílian. O perfil do gestor desportivo: um estudo nos Centros de Esporte e Lazer da Prefeitura Municipal de Manaus.2011. 111 f. Dissertação (Mestrado) - Faculdade de Motricidade Humana, Universidade Técnica de Lisboa, Lisboa, 2011. 